\title{
Assessing the Quality Level of Corn Tortillas with Inductive Characterization and Digital Image Analysis"
}

\author{
Marco A. Moreno-Armendáriz, Salvador Godoy-Calderon, \\ Hiram Calvo, and Oscar M. Rojas-Padilla \\ Centro de Investigación en Computación, Instituto Politécnico Nacional, \\ AV. Juan de Dios Bátiz S/N, Unidad Profesional "Adolfo López Mateos", \\ México, D. F. C. P. 07738, México \\ mam.armendariz@gmail.com, sgodoyc@gmail.com, \\ hcalvo@cic.ipn.mx, oscarrjs@gmail.com
}

\begin{abstract}
Characterization and classification of corn tortillas turns out to be an extremely delicate and difficult process when dealing with regulations for import/export and production process certification. In this paper we present a method for non-invasive feature extraction, based on digital imaging and a series of procedures to characterize different qualities of corn tortillas for their later classification. The novelty in this whole method lies in the extremely reduced set of features required for the characterization with only geometrical and color features. Nonetheless, this set of features can assess diverse quality elements like the homogeneity of the baking process and others alike. Experimental results on a sample batch of 600 tortillas show the presented method to be around $95 \%$ effective.
\end{abstract}

Keywords: Inductive characterization, digital image analysis, corn tortilla.

\section{Introduction}

Tortillas are a fundamental element in the diet of Mexican people. Tortillas have been hand-made since ancient times. As a result of the increasing demand of this product, even from other countries, new production methods have become a necessity for massively producing tortillas; their quality must be assessed to comply with industrial production standards as well as import/export regulations [17]. It has been shown $[12,9]$ that sensory characteristics are of great importance for consumers, however, for small and mid-size producers there is currently no protocol for quality-control; there is an absence of standards and guides to evaluate the sensory impression of consumers, i.e., color, shape, spots, etc., or some other kind of imperfections resulting from their elaboration and baking process, and thus, tortillas are evaluated manually resulting in the fact that the quality of the tortilla is not uniformly assessed.

\footnotetext{
* The authors wish to thank the support of the Mexican Government (CONACYT, SNI,
} SIP-IPN, COFAA-IPN, and PIFI-IPN). 
In this work we propose to classify tortillas using digital image analysis, a noninvasive sensor that does not cause damage to the produce.

In Section 2 we describe the techniques used for extracting the features used for tortillas classification; in Section 3 we describe our method of inductive classification; in Section 4 we show results and discussion, and finally we draw our conclusions.

\section{Feature Extraction}

In this section we will introduce the methods we use for classifying tortillas. Sample tortillas will be taken from three different sources: small producer, supermarket and industrialized. To begin, a digital image of each side of the corn tortilla is taken. See Figure 1.

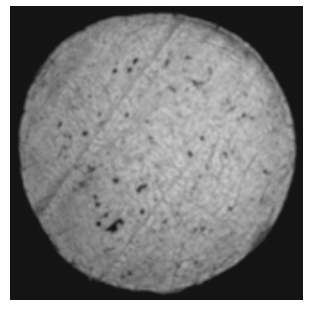

(a)

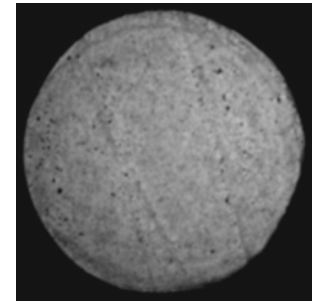

(b)

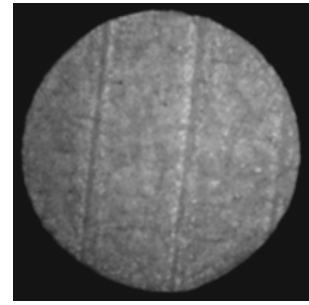

(c)

Fig. 1. Tortillas sampled from: (a) small shop, (b) supermarket, (c) industrial batch

Geometric and color features are extracted from a sample of corn tortilla images to determine the quality level of the tortilla. Geometric features include curvature, symmetry and border deformations, as well as the presence of some defects like ruptures, holes or bends. Color features include dark/bright spots, color homogeneity and average color.

\subsection{Color Features}

Each image is separated into Red, Green and Blue channels (RGB), and then three interest areas are determined for each image by color-reducing each channel. Those areas bring evidence of the cooking degree on the tortilla surface. A tortilla can be considered raw (undercooked), well cooked or burnt (excessively cooked) by counting the surface area occupied by bright spots, average color spots or dark spots respectively.

Since images have a color-depth of eight bits, every pixel within each channel holds an intensity value in the range $[0,255]$. To reduce the number of intensity levels in an image channel, we establish a threshold in the following manner: each pixel's intensity value is mapped to one of three pre-determined levels by two thresholds. If the intensity level of the pixel reads under 30, then it is mapped to a zero value 
(pure black), if it reads any value between 31 and 180, then it is mapped to a value of 64 (mid-value color) and finally, if it reads any value between 181 and 255, it is mapped to a value of 128(high value). These thresholds were determined based on the tortilla's image histograms, so that the areas of interest are successfully extracted. Background pixels are also mapped to a zero-intensity value, so that they appear to be part of the dark spots area, but they are filtered out during the color-features extraction process, see Figure 2. In this Figure $f_{1}$ to $f_{8}$ are the features used for the classification process described in Section 3.

Once the three interest areas within each image are selected, the following processes take place: contour identification, tortilla surface area filtering and edge characterization; then, features are extracted.

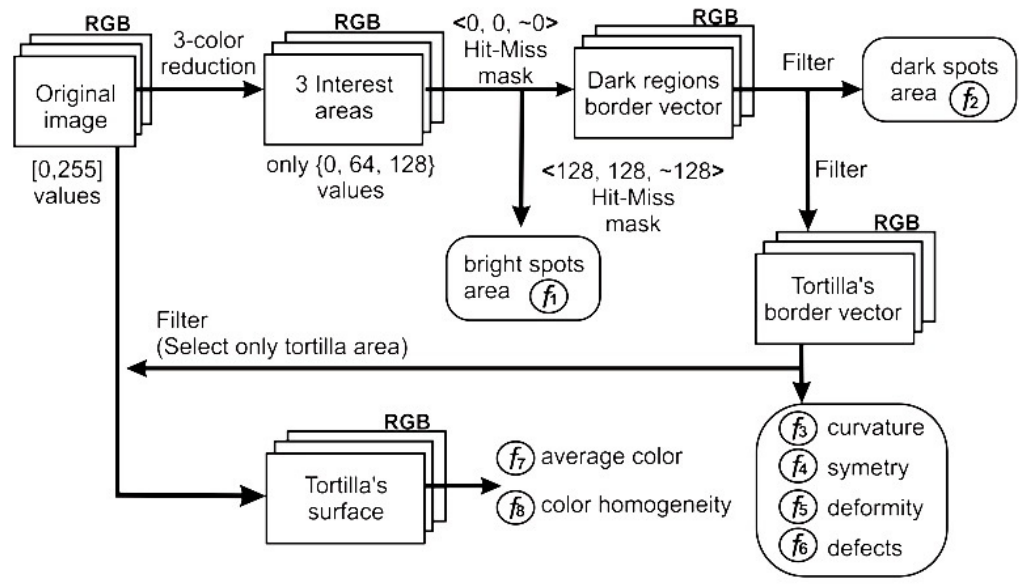

Fig. 2. Image processing process for feature extraction
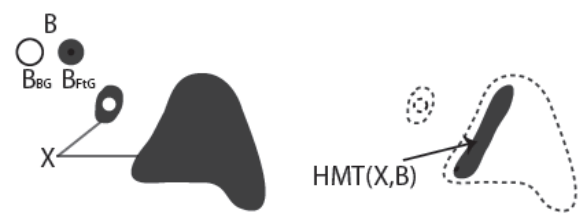

Fig. 3. An example of a hit-or-miss transformation. It shows that it is necessary for both reference points to meet at the desired target for the operation to be performed, otherwise a gap appears in the region of the resulting image (Taken from [19]).

Contour Identification. Two morphological hit-or-miss operators (see Figure 3) are proposed. Each structure element is applied four times, rotating $90^{\circ}$ each time, and then we use the union of the results. The first structure element, composed by the values $\mathrm{B}_{\mathrm{N} 1}=\{0, \neg \mathbf{0}, \neg 0\}$ looks for image regions matching one black pixel (the background) followed by two consecutive non-zero values (the tortilla surface). The second structure element $\mathrm{B}_{\mathrm{C} 1}=\{\neg 128, \neg \mathbf{1 2 8}, 128\}$ looks for regions matching 
two consecutive non high-intensity pixels followed by a high-intensity pixel, thus, finding raw areas inside the tortilla. See Figure 4.

Excessively cooked regions on the tortilla surface appear as dark spots on the digital image, while raw or undercooked regions appear as bright spots. Both kinds of spots can have varying sizes as can be seen on Figure 5.

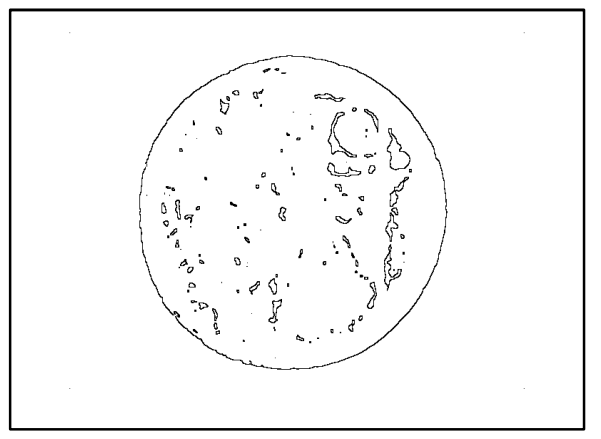

(a)

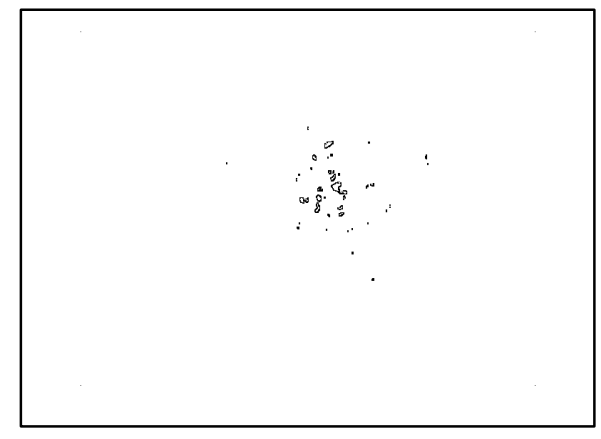

(b)

Fig. 4. Result of applying the structure element $B_{N 1}=\{0, \neg 0, \neg 0\}$ (a) and $B_{C 1}=\{\neg 128, \neg 128$, $128\}$ (b) on one sample

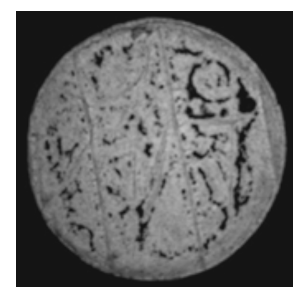

(a)

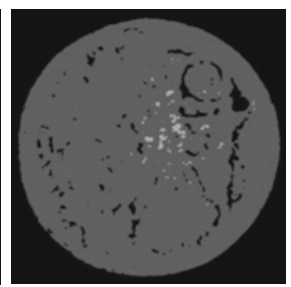

(b)

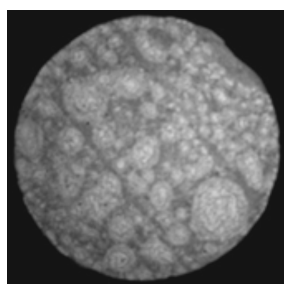

(c)

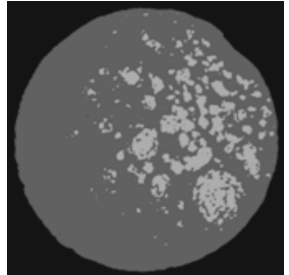

(d)

Fig. 5. (a) Tortilla with dark spots from excessively cooked regions (b) interest regions from the same tortilla; (c) Tortilla with bright spots from raw regions (d) interest regions detected.

We propose the following color features:

Color homogeneity (cHom) describes a uniform temperature distribution during the cooking process and, of course, the average color depends on the corn type the tortilla is made of. A correctly baked tortilla must show a uniform specific color with no darker or brighter regions. A raw or incompletely baked tortilla will show some brighter regions, while a burned or excessively baked one will show some darker ones. Color will be less homogeneous in the last two cases.

Average Brightness ( Lavg). This feature identifies, as the name implies, the amount of light from the surface, which ideally should be equivalent throughout the surface. This value varies depending on the type of dough used for making the product and may go from dark yellow hues, to whitish yellow. This parameter is obtained by the average of all pixels' color value contained in the tortilla surface. 
Variance of light intensity (Lvar). The non-homogeneous colouration on the surface is caused by burnt or raw areas and poorly processed corn leftovers. These defects cause the brightness of the surface not to be homogeneous, which may vary dramatically from one pixel to another. In the ideal case, the variance should be zero, so that for a uniform brightness a value close to zero would be expected. For the calculation of this feature, as with the average brightness, all pixels of the surface are used and then the variance of these values is used.

Burned areas (burntA). Burned areas represent sections of varying sizes of burnt dough produced by an excess of cooking. Coloring of these areas is common to any hue of the tortillas, making it easy to identify. For its identification we use the border extracted as described previously with the hit-miss transformation, by filtering the edges of the burned areas from the tortilla edges. Obtaining this feature is done in an indirect way, because by counting the edge borders we infer that as there are more edge pixels, the size of the area would be grater.

Raw areas $(\boldsymbol{r a w A})$. The raw areas represent sections of dough that did not reach the proper cooking and have a lighter color than the rest of the surface. As with the burnt areas, the extraction of the obtained raw areas is obtained from the surface segmentation, and calculation is carried out indirectly by counting the pixels of the edges.

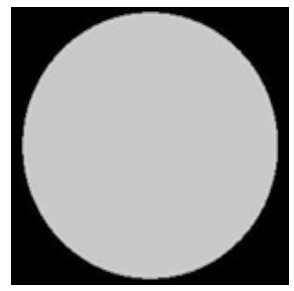

(a)

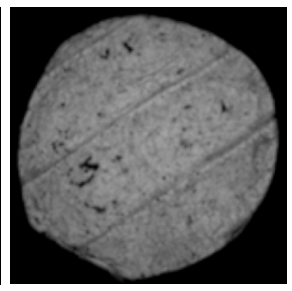

(b)

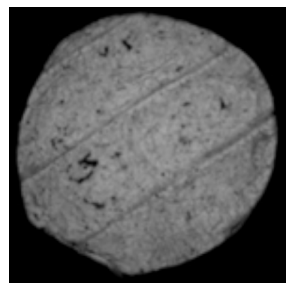

(c)

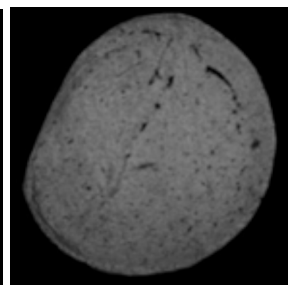

(d)

Fig. 6. (a) Model of a perfect tortilla, (b) tortilla without symmetry, (c) tortilla with a slight defect along its border, (d) tortilla with a large defect along its border

\subsection{Geometric Features}

In order to get a discrete representation for the tortilla's border, we divide it into 64 arc segments. Each arc is projected over the horizontal axis and the length of the projection is measured $[22,2]$. A characteristic descriptor for each tortilla side is made up with the lengths of the 64 projected arcs [8].

Geometry-related features aim to capture those attributes related to the shape of the tortilla including curvature, symmetry and continuity. According to export regulations, a high quality tortilla must have a nearly perfect circular shape with no holes and no bends or breaks along its border (See Figure 6). Since a discrete characteristic chain is used to describe the border of the tortilla, this chain is compared with the corresponding chain of a perfect circle as described in [7] (see Figure 7). If both chains are identical then the tortilla border has the best quality available. The following features were extracted: 

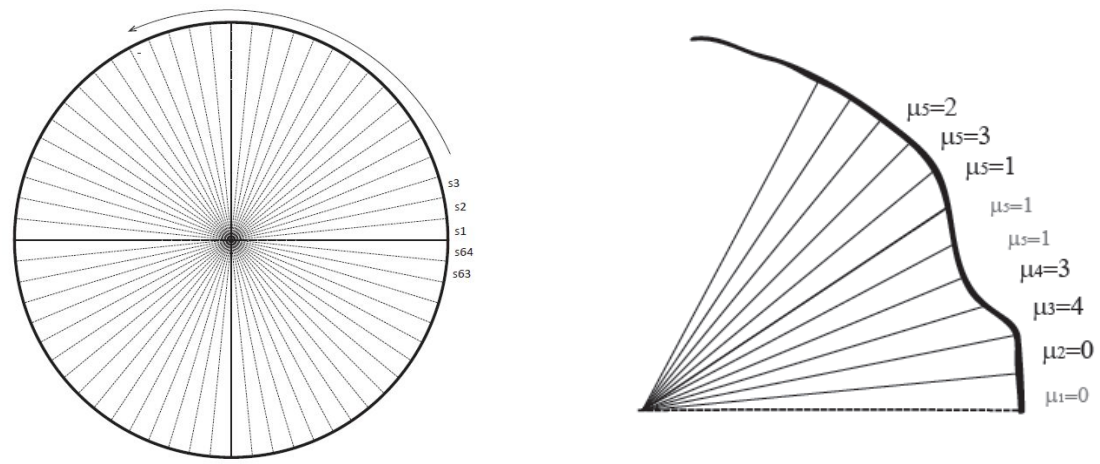

Fig. 7. Projected arcs for measuring the 'roundness' of tortilla.

A defect is shown to the right.

Defects $(\boldsymbol{d} f \boldsymbol{c t})$. A defect in a tortilla occurs when a small irregularity appears on the edge, perceptibly altering the circularity of the edge section. This change is often abrupt and has a short distance of no more than one centimeter (no more than 1/8 of the circumference.) Convexity is affected.

Deformations (dfrm). The deformation in a tortilla occurs where one segment has a non-circular tendency. In this segment the edge is straight (no more than $1 / 4$ of the circumference.) Convexity is not affected.

Circularity (circ). Its value is calculated by adding up the differences of each one of the diameters with regard to an average.

\section{Inductive Classification}

One of the most common techniques used to obtain the rules of inductive learning is known as "divide and conquer" [5]. This technique, which appeared in the early 80's, is named after the method applied to construct a rule induction, dividing the initial set of knowledge rules and selecting the rules that provide better coverage rates. Important works using this technique are Michaslki [14, 16], Clark [3], and Rivest [18]; several innovations and/or adaptations of the techniques proposed by them arose for nearly a decade after the publication of these mentioned works.

One of these works to improve learning technique was performed by the same Michalski in the mid-1980s. The STAR method is based on the principle of "divide and conquer" and it further allowed the resolution of everyday problems or applications that have large numbers of possible solutions. Finally it emerged and positioned itself as the BOUNDSTAR and REDUSTAR methodologies (see [13] for an in-depth explanation). 


\subsection{Tortilla Characterization by Inductive Learning}

Once the features for all samples are defined and calculated, we reach the stage of inductive learning where the characterizing rules are learnt. The learning process is based on the model of the BOUNDSTAR algorithm [13] that seeks to obtain a characterization from events calculated by parameterizing features, with the conjunctions of these, trying to get a generalization and thereby obtaining rules which shape knowledge and allow characterization. The last step of this process is to implement the rules to a new group of samples and the evaluation of the classification results are obtained.

\subsubsection{Parameterization of Features}

Each dataset of characteristics, both color and texture and geometric data has a different distribution for each class along each feature domain. Based on this premise, the parameterization is performed for each set of data for a range in which each of the classes is covered in greater proportion with the rest of them, i.e., this class has a percentage of maximum coverage in this range. Thus, $r$ will be obtained for each value of each feature, being $r$ the number of classes that we seek to characterize.

The set of all observed events will form an initial knowledge base. Although not all of those may be able to completely cover any class above the others. Some of the features contained in this knowledge base will be discarded, as they are of marginal use for the creation of knowledge rules, since their coverage percentage is very strong on some classes, and very weak for other classes at the same time.

Each event is calculated relying on the histogram of each feature as well. Figure 8 shows the distribution of the feature Lvar. We can see that the class 2 (asterisk symbol) will get high percentage of coverage and a considerable difference with respect to the percentage of coverage of other classes, a situation that does not happen for Class 3 (circular symbol) and even less for Class 1 (square symbol), because the samples of these classes are overlapping.

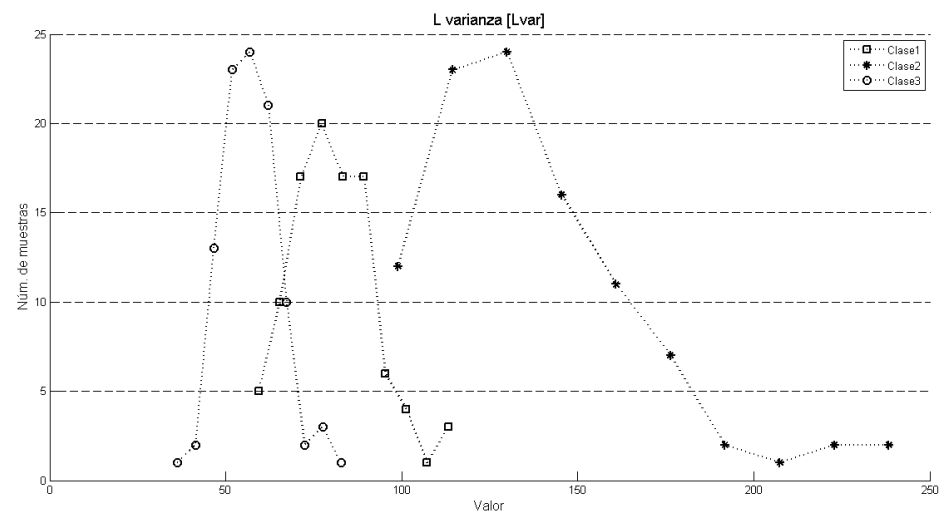

Fig. 8. Lvar feature histograms. As noted, it is possible to achieve a separation of the class 2 which intersects only in a small range with samples of class 1 . 
An event, either from the original knowledge base or from a later one, can be considered, like in the case of a complex feature, stronger than any other characterization if it achieves the characterization of all the elements of a class under parameters which only the elements of this class meet, while none of the remaining classes' elements meet. So if we happen to have an event that accomplishes these goals, then this event is considered the strongest possible characterization for the elements of the class, and may even be considered as a stop condition for the algorithm.

\section{$4 \quad$ Experimental Results}

We obtained a sample of 600 tortillas, from three kinds of commercial establishments (see Figure 1), 200 from each kind. With help from some experts in food engineering, a model of the perfect tortilla made from each possible type of corn was defined (see Figure 6) and then we proceeded to apply the induction classification. We learned from the first half of 300, and then we evaluated with the remaining 300. The extraction of the first two color features of the 300 images of tortilla during the learning phase can be seen in Figure 9. In the case of Lavg, it can be seen that the data of class 2 are distant with respect to the other classes. This phenomenon is generated by the use of different materials (different masses or corn flour) which impact directly on the color and brightness of the product. Furthermore, although Lvar and Lavg are directly related, the distribution of such data is not necessarily the same. As shown in Figure 9, the variance of color (light) samples among those of the same class can be wider. For both features at least one class is easily identifiable above the rest; this will be an advantage for the parameterization and search of characteristic features of each class.

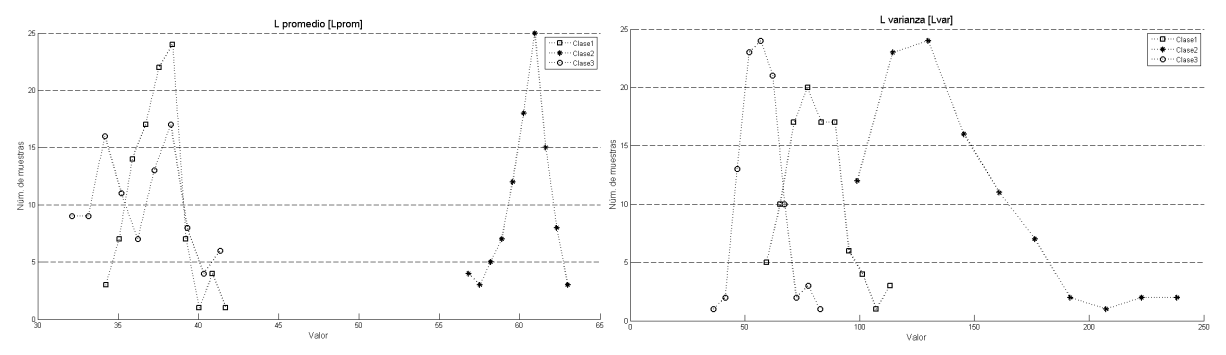

Fig. 9. The first graph shows the data distribution of the three different classes with regard to the average $\mathrm{L}$

The other two color characteristics burntA and rawA are shown in Figure 10. There it can be seen that for both features the three classes have a poor dispersion, so that a differentiation between samples of a particular group will not be easy, or even impossible to achieve because there is little scatter in the data. Then, this feature is not considered as very important for the classification. 

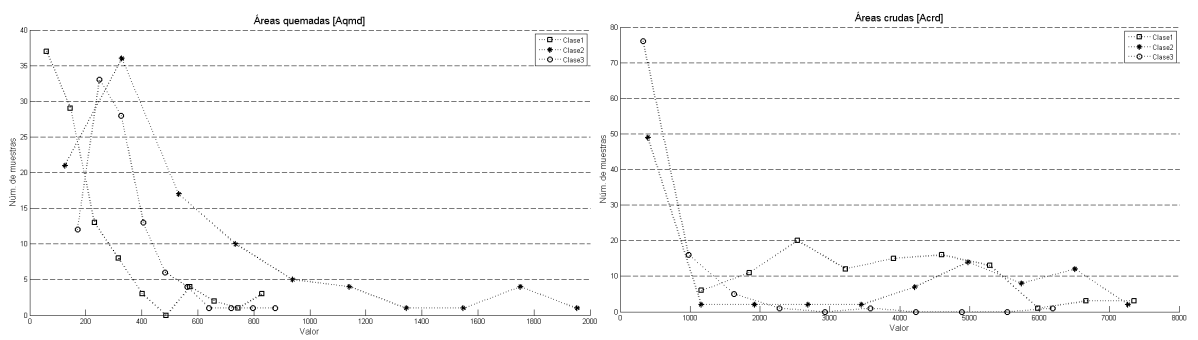

Fig. 10. The first graph shows the distribution of the burntA data, which the lower one represents $\operatorname{rawA}$; both show a difficult separation of these classes

With regard to the geometrical features, the results extracted from the images can be seen in Figure 11 and Figure 12. $d f c t$ seems to provide good information for characterization, while as defm and circ exhibit little disjoint accumulation. A further analysis of the distribution and these results is offered in the discussion of results section.
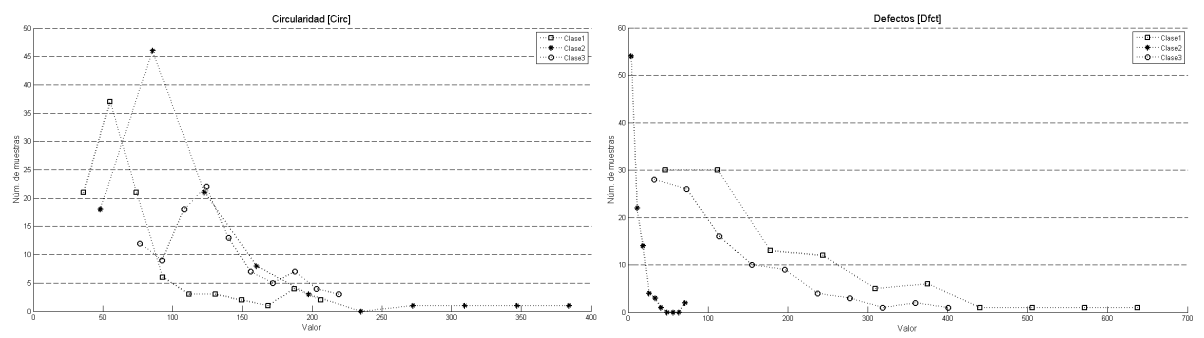

Fig. 11. The circularity circ (pictured left), presents some difficulty differentiating classes, on the contrary, the defects $d f c t$ (right) samples differentiate one class above the rest

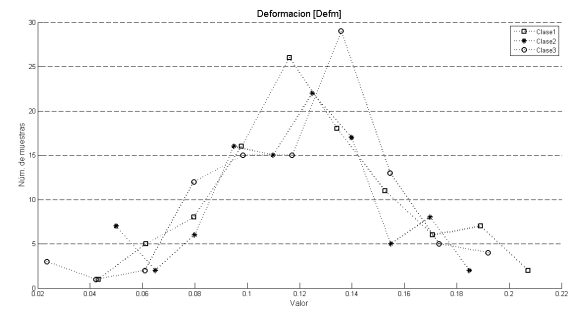

Fig. 12. The distribution of strain (defm) in the samples of the three classes presents difficulties for the separation of samples

Other features such as correlation, homogeneity, and entropy distribution are not shown, since they were are not considered in the process of inductive learning. 


\subsection{Characterization}

Once we have the data shown in the histograms above, in order to obtain knowledge rules using inductive learning, it is necessary to first perform the parameterization of the extracted features.

Because the inductive learning method used is BUNDSTAR, and that this is based on a set of events, it is necessary first to define events together. These events will form the initial knowledge base. Table 1 shows the coverage results of these events. The best percentage of coverage obtained for the each class of the feature is highlighted in bold. From Table 5 we can see some interesting phenomena in covering classes:

1. The Lavg complex feature under the proposed parameters, is the strongest possible because it manages to strongly characterize to class 2 and thus creates two disjoint sets. The first is that all the elements of the class 2 and the other is formed by all elements in classes 1 and 3 .

2. The Lvar complex feature can characterize two different classes, using different parameters, classes 2 and 3, being stronger characterization of class 2 .

3. The circ complex feature, despite all samples characterize class 3 does not achieve it in a convenient way, since $88 \%$ of characteristic samples of class 2 are characterized too.

4. The complex feature $d f c t$ can almost perfectly characterize the class 2 .

5. There are other features that were not included in Table 5 because the coverage percentages are not suitable, for example, the feature called $d f r m$ is not strong enough to characterize a class because the difference is minimal.

Table 1. Coverage pct. of the proposed complex features from the learning stage

\begin{tabular}{clccc}
\hline$\#$ & Complex feature & Class1 & Class 2 & Class 3 \\
\hline 1 & Lavg $>50$ & 0 & $\mathbf{1 0 0}$ & 0 \\
2 & Lvar $>91$ & 17 & $\mathbf{1 0 0}$ & 0 \\
3 & Lvar $<85.5$ & 67 & 0 & $\mathbf{1 0 0}$ \\
4 & burntA $<220$ & $\mathbf{7 0}$ & 21 & 14 \\
5 & $220<$ burntA $<450$ & 19 & 37 & $\mathbf{7 2}$ \\
6 & rawA $>1200$ & $\mathbf{9 6}$ & 49 & 10 \\
7 & circ $>60$ & 48 & 88 & $\mathbf{1 0 0}$ \\
8 & dfct $<45$ & 9 & $\mathbf{9 8}$ & 21 \\
9 & $0.3262<$ dfrm $<0.4452$ & $\mathbf{1 0 0}$ & 2 & 37 \\
\hline
\end{tabular}


Table 2. Set of solutions that characterize better coverage percentages classes. The rules to be used in the generalization are shown in bold.

\begin{tabular}{clcccc}
\hline Class & \multicolumn{1}{c}{ Solution set } & C 1 & C 2 & C 3 \\
\hline \multirow{3}{*}{$1 \quad$ burnt $A<220 \wedge$ circ $<60 \wedge$ Lavg $<50$} & 38 & 4 & 0 \\
& rawA $>\mathbf{1 2 0 0} \wedge$ Lavg $<\mathbf{5 0}$ & $\mathbf{9 6}$ & $\mathbf{0}$ & $\mathbf{1 0}$ \\
\multirow{3}{*}{2} & $0.3262<d$ frm $<0.4452 \wedge$ Lavg $<50$ & 52 & 0 & 0 \\
\multirow{2}{*}{3} & Lavg $>\mathbf{5 0}$ & $\mathbf{0}$ & $\mathbf{1 0 0}$ & $\mathbf{0}$ \\
& Lvar $<85.5 \wedge$ & $0.3262>d$ frm $<0.4452$ & 0 & 0 & 63 \\
& $220<$ burntA $<450 \wedge$ Lvar $<91$ & 15 & 0 & 72 \\
& Lavg $<\mathbf{5 0} \wedge$ rawA $<\mathbf{1 2 0 0} \wedge$ circ $>\mathbf{6 0}$ & $\mathbf{0}$ & $\mathbf{0}$ & $\mathbf{9 0}$ \\
\hline
\end{tabular}

Table 3. Coverage percentages obtained using complex features after re-learning

\begin{tabular}{lccc}
\hline \multicolumn{1}{c}{ Learning Rules } & C 1 & C 2 & C 3 \\
\hline Lavg $>50$ & 0 & 100 & 0 \\
$(\operatorname{Lavg}<50)^{\wedge}(\operatorname{rawA}>1200)$ & 96 & 0 & 10 \\
$(\operatorname{Lavg}<50)^{\wedge}(\operatorname{rawA}<1200) \wedge(\operatorname{circ}>60)$ & 0 & 0 & 90 \\
\hline
\end{tabular}

\subsection{Evaluation}

To validate the proposed rule of knowledge, we used the 300 remaining samples. As mentioned in the previous section, it is possible that during the classification of new samples some of them are not classified in any of the three classes defined. These phenomenon occurs when the samples in question are outside of the parameters of the features and then they can be considered as out of context objects.

The results of the classification of the new group of samples is shown in Table 4 .

Table 4. Classification results of 300 new samples using complex features listed in Table 2

\begin{tabular}{ccccc}
\hline & Class 1 & Class 2 & Class 3 & Not classified \\
Classified Samples & 84 & 100 & 113 & 3 \\
\hline
\end{tabular}

Table 5. Classified samples. The rows are read the actual class and columns are the class to which they were assigned according to their features and the learnd rules of knowledge.

\begin{tabular}{cccccc}
\hline & & \multicolumn{4}{c}{ Classification } \\
& & Class 1 & Class 2 & Class 3 & No class \\
\hline \multirow{2}{*}{ Real } & Class 1 & 82 & 0 & 16 & 2 \\
Class & Class 2 & 0 & 100 & 0 & 0 \\
& Class 3 & 2 & 0 & 97 & 1 \\
\hline
\end{tabular}


Table 4 shows that there are samples which are not classified correctly by the learnt rules. This is evident if we see the class 3 , which has more samples that it might contain. For validating the results we need to determine to which class the samples really belong, and to which class they were assigned due to their properties. This confusion-matrix is shown in Table 5.

\subsection{Discussion of Results}

The results of the characterization of the three classes proposals can be seen in Table 1 , where the percentages of coverage are achieved by early complex features. From this learning stage it can be appreciated, as anticipated in the extraction of features, that not all features that are useful for the characterization, however, there are complex features capable of characterizing a class above the other with excellent results. Examples are traits $L a v g>50$ and Lvar> 91, which are close to $100 \%$ coverage in the class of interest, class 2.

After the implementation of the BOUNDSTAR algorithm, we obtained the ruleset presented in Table 2. This ruleset represents the best solutions for each class and it can be used independently for different purposes.

To validate the learnt rules, we evaluate their performance and determined the following values: memory, precision, specificity and accuracy for each of the classes determined, these results are:

1. The rule for class 1 (tortillas package) is: $(\operatorname{Lavg}<50)^{\wedge}(\operatorname{rawA}>1200)$ having an accuracy of $93 \%$, a precision of $97 \%$, a specificity of $99 \%$ and $82 \%$ memory; this latter being affected mainly by the 18 samples that were classified in another class.

2. The rule that characterizes the class 2 (supermarket tortillas) is $L a v g>50$, this rule has the distinction of being an ideal case, it is a strong rule. This property can be seen in the histogram of Lavg, see Figure 8; this graph shows the classes 1 and 3 separated by a wide range of values with respect to Class 3 , this means that visually the samples of class 2 are much clearer and this is enough to characterize.

3. The characterization rule for class 3 (tortilla from a neighborhoodstore) is: (Lavg $<50)^{\wedge}($ rawA $<1200) \wedge(\operatorname{circ}>60)$, which has an accuracy of 93\%, Memory $97 \%$, specificity of $92 \%$ and a precision of $85 \%$. This low precision is due to the 16 samples of Class 1 that are classified in this class. This problem is due to the similarity of its luminosity. In this case use the circularity is used for distinguishing these two kinds of samples; however this is obtained yielding a lower precision with regard to that of the other classes.

\section{Conclusions}

We have found that the inductive characterization of three classes of producers is achieved with high percentages of coverage and precision; particularly in the case of 
Group 2 with a coverage of $100 \%$. Importantly, despite the diversity of variables involved in making tortillas, it was possible to find distinctive patterns of each manufacturer in production. The proposed color and geometric features were useful for achieving this classification. The most important features in the classification, given the high percentage of coverage for the desired classes were color features. Significantly, color features become extremely important in characterizing when considering the preference of the consumer.

In general, we could say that it is possible to make a quantification of the visual features of tortillas, using only images, and using these features to obtain an inductive characterization of different producers. This is shown in the results of Table 2, since the exposed ruleset characterizations represent the best for each class, and they are themselves a numerical representation of the visual properties of each class of common tortillas. Despite the good results obtained by the learned knowledge rule, characterization results could be improved by using not exclusively visual attributes, but also considering other features resulting from a biochemical analysis of the samples, such as moisture, density, elasticity, among others. This latter is left as a future work.

\section{References}

1. Adams, J., Parulski, K., Spaulding, K.: Color processing in digital cameras. IEEE Micro. 18(6), 20-30 (1998)

2. Cai, W., Yu, Q., Wang, H.: A fast contour-based approach to circle and ellipse detection. In: Fifth World Congress on Intelligent Control and Automation, vol. 5, pp. 4686-4690 (2004)

3. Clark, P., Tim, N.: The CN2 induction algorithm, Tahoe City,CA (1989)

4. Cortés-Goméz, A., Martín-Martínez, E.S., Martínez-Bustos, F., Vázquez-Carrillo, G.M.: Tortillas of Blue Maize (zeamays 1.) Prepared by a Fractioned Process of Nixtamalization: analisys using response surface methodology. J. of Food Eng. 60(3), 273-281 (2001)

5. Domingos, P.: Unifying Instance-Based and Rule-Based Induction. Machine Learning 24(2) (1996)

6. Gonzalez, R., Woods, R.: Digital Image Processing, 3rd edn. Prentice Hall, Pearson (2008)

7. Gupta, L., Srinath, M.D.: Contour Sequence Moments for the Classification of Closed Planar Shapes. Pattern Recognition 20(3), 267-272 (1987)

8. Hastie, T., Tibshirani, R., Friedman, J.: The Elements of Statistical Learning: Data Mining, Inference, and Prediction. Springer (2003)

9. Herrera-Corredor, J., Saidu, J., Khachatryan, A., Prinyawiwatkul, W., Carballo-Carballo, A., Zepeda-Bautista, R.: Identifying Drivers for Consumers Acceptance and Purchase Intent of Corn Tortilla. J. of Food Sc. 72(9), 727-730 (2007)

10. Ibarra-Manzano, M.A., Devy, M., Boizard, J.L.: Real-time classification based on color and texture attributes on an FPGA-based architecture. In: Conference on Design and Architectures for Signal and Image Processing, pp. 250-257 (2010)

11. Malamas, E.N., Petrakis, E.G.M., Zervakis, M., Petit, L., Legat, J.D.: A Survey on Industrial Vision Systems, Aplications and Tools. Image and Vision Computing 21(3), $171-188$ (2003) 
12. Mery, D., Chanona-Pérez, J.J., Soto, A., Aguilera, J.M., Vélez-Rivera, N., ArzateVázquez, I., Gutiérrez-López, G.F.: Quality Classification of Corn Tortillas using Computer Vision. J. of Food Eng. 101, 357-364 (2010)

13. Michalski, R.S., Stepp, R.: Automated Construction of Classifications: Conceptual Clustering versus Numerical Taxonomy. IEEE Trans. on Pattern Analysis and Machine Intelligence PAMI-5(4), 396-410 (1983)

14. Michalski, R.S.: A theory and methodology of inductive learning 20 (1983)

15. Michalski, R.S., Chilausky, R.L.: Learning by being told and learning form examples: an experimental comparison of the two methods of knowledge adquisition in the context of developing an extra system for sorbeandesease diagnosis. Policy Analysis and Information Systems 4(2), 125-160 (1980)

16. Michalski, R.S., Mozetic, I., Hong, J., Lavrac, N.: The multi-purpose incremental learning system AQ15 and its testing application to three medical domains. In: Proceedings of the Fifth National Conference on Artificial Intelligence, Philadelph (1986)

17. Norma-Oficial-Mexicana, NOM-187-SSA1/SCFI-2002: Productos y servicios. Masa, tortillas, tostadas y harinas preparadas para su elaboración y establecimientos donde se procesan. Especificaciones sanitarias. Secretaría de Economía, Estados Unidos Mexicanos (2003)

18. Rivest, R.L.: Learning decision lists. Machine Learning, 229-246 (1987)

19. Soille, Morphological Image Analisys. Springer (2004)

20. Tahir, M.A., Bouridane, A.: An Fpga Based Coprocessor for Cancer Classification Using Nearest Neighbour Classifier. In: IEEE International Conference on Acoustics, Speech and Signal Processing, vol. 3, pp. III-1012-III-1015 (2006)

21. Terasic Inc., http: / / www. terasic. com.tw

22. Watanabe, T., Matsumoto, M.: Recognition of Circle Form Using Fuzzy Sequential System. In: Twenty-First International Symposium on Multiple-Valued Logic, pp. 85-92 (1991) 